\title{
Dielectric properties of amorphous phase-change materials
}

\author{
C. Chen, ${ }^{1}$ P. Jost, ${ }^{1}$ H. Volker, ${ }^{1}$ M. Kaminski, ${ }^{1}$ M. Wirtssohn, ${ }^{1}$ U. Engelmann, ${ }^{1}$ K. Krüger, ${ }^{1}$ F. Schlich, ${ }^{1}$ \\ C. Schlockermann, ${ }^{1}$ R. P. S. M. Lobo, ${ }^{3,4}$ and M. Wuttig ${ }^{1,2, *}$ \\ ${ }^{1}$ I. Physikalisches Institut (IA), RWTH Aachen University, 52056 Aachen, Germany \\ ${ }^{2}$ JARA-Institut Green IT, JARA-FIT, Forschungszentrum Jülich GmbH and RWTH Aachen University, 52056 Aachen, Germany \\ ${ }^{3}$ LPEM, ESPCI Paris, PSL Research University, CNRS, 10 rue Vauquelin, 75005 Paris, France \\ ${ }^{4}$ Université Pierre et Marie Curie, Sorbonne-Universités, 75005 Paris, France \\ (Received 22 August 2016; revised manuscript received 8 October 2016; published 14 March 2017)
}

\begin{abstract}
The dielectric function of several amorphous phase-change materials has been determined by employing a combination of impedance spectroscopy $(9 \mathrm{kHz}-3 \mathrm{GHz})$ and optical spectroscopy from the far- $\left(20 \mathrm{~cm}^{-1}\right.$, $0.6 \mathrm{THz})$ to the near- $\left(12000 \mathrm{~cm}^{-1}, 360 \mathrm{THz}\right)$ infrared, i.e., from the DC limit to the first interband transition. While phase-change materials undergo a change from covalent bonding to resonant bonding on crystallization, the amorphous and crystalline phases of ordinary chalcogenide semiconductors are both governed by virtually the same covalent bonds. Here, we study the dielectric properties of amorphous phase-change materials on the pseudobinary line between $\mathrm{GeTe}$ and $\mathrm{Sb}_{2} \mathrm{Te}_{3}$. These data provide important insights into the charge transport and the nature of bonding in amorphous phase-change materials. No frequency dependence of permittivity and conductivity is discernible in the impedance spectroscopy measurements. Consequently, there are no dielectric relaxations. The frequency-independent conductivity is in line with charge transport via extended states. The static dielectric constant significantly exceeds the optical dielectric constant. This observation is corroborated by transmittance measurements in the far infrared, which show optical phonons. From the intensity of these phonon modes, a large Born effective charge is derived. Nevertheless, it is known that crystalline phase-change materials such as GeTe possess even significantly larger Born effective charges. Crystallization is hence accompanied by a huge increase in the Born effective charge, which reveals a significant change of bonding upon crystallization. In addition, a clear stoichiometry trend in the static dielectric constant along the pseudobinary line between GeTe and $\mathrm{Sb}_{2} \mathrm{Te}_{3}$ has been identified.
\end{abstract}

DOI: 10.1103/PhysRevB.95.094111

\section{INTRODUCTION}

Chalcogenide-based phase-change materials (PCMs) are very attractive due to their potential for optical and electrical data storage applications [1-4]. These applications rely on the pronounced property contrast between the amorphous and the crystalline phase in combination with the possibility to switch rapidly between these phases. In recent years, electronic phase-change random access memories (PRAM) have raised particular interest since they enable data storage attributes, which differ from those of the presently employed Flash and dynamic random access memories (DRAM). In particular, PRAMs uniquely combine nonvolatility and high switching speeds (10 ns) [5] with good endurance $\left(10^{10}\right.$ cycles) [6].

In the last decade, substantial progress has been made in understanding structure, bonding, and charge transport in crystalline PCMs [7-11]. At the same time, the resistance drift [12] in the amorphous state, the high switching speeds, and the fact that amorphous PCMs are non-Zachariasen glasses [13] have motivated significant interest in the atomic arrangement of the amorphous state. Hence, $a b$ initio theory as well as advanced x-ray and neutron methods have been employed

\footnotetext{
*wuttig@physik.rwth-aachen.de

Published by the American Physical Society under the terms of the Creative Commons Attribution 4.0 International license. Further distribution of this work must maintain attribution to the author(s) and the published article's title, journal citation, and DOI.
}

extensively [14-16]. Nevertheless, as can be inferred from the ongoing discussion [17,18], the atomic arrangement and bonding in the amorphous state as well as the phase-change mechanism are still under debate. Consequently, the already mentioned amorphous-state phenomena resistance drift and threshold switching [19] are not yet fully understood, which hampers the development of PCM-based electronic devices and the realization of multilevel memories [20].

This paper presents a comprehensive study of the subband-gap dielectric properties of amorphous PCMs. Our experiments investigating polarizability and phonon frequencies come from a completely different direction from the aforementioned advanced $\mathrm{x}$-ray and neutron studies, but they are sensitive to and therefore shed light on the very same issues: (local) atomic arrangement and bonding in the amorphous network. In that, our data can be regarded as complementary to these studies. Detailed knowledge of the dielectric response between the DC limit and the infrared regime provides valuable input to many of the questions currently under debate: For instance, a plethora of theoretical models for the structure of amorphous PCMs has been developed [21-24]. As the polarizability depends on subtle details of structure and bonding, knowledge of the static dielectric constant and the phonons can help in gauging and singling out competing density functional theory (DFT) models for amorphous PCMs. In addition, exact knowledge of the static dielectric constant is desirable for the simulation of capacitive effects in high-speed PRAM devices [25].

Moreover, the static dielectric constant provides valuable insights as to the bonding mechanism in amorphous PCMs: In 
ordinary covalently bonded semiconductors, the same bonding mechanism prevails in the amorphous and the crystalline state. This is very different in PCMs, where resonant bonding exists in the crystalline $[7,8]$ but not in the amorphous state $[11,26,27]$. The nature of the bond has an effect on the polarizability of the electron system (optical dielectric constant), the polarizability of the phonons (static dielectric constant), and, of course, the phonon frequencies. These quantities can easily be derived from the dielectric function. Hence, comparing differences in the dielectric function of amorphous and crystalline PCMs of the same stoichiometry should help in unraveling the bonding in amorphous PCMs.

Similar to the already mentioned competing structure models, several mutually incompatible models for the charge transport in amorphous PCMs, such as the small polaron model [28], the Poole-Frenkel model [29], and the band transport model [30], have been suggested. Knowing the frequency dependence of the conductivity can help to determine the charge transport mechanism.

Interestingly, only very few attempts to determine the static dielectric constant have been reported. The conclusions derived from these earlier investigations are puzzling. Both Prokhorov et al. [31] and Santos et al. [32] performed measurements up to the $\mathrm{MHz}$ range. They report numbers of the static dielectric constant $\varepsilon_{\text {st }}$ (see remark regarding the notation [33]), which are, within error bars, identical to the high-frequency (optical) dielectric constant $\varepsilon_{\infty}$ as derived by [8]. This equality of $\varepsilon_{\text {st }}$ and $\varepsilon_{\infty}$ is difficult to understand. For any material possessing optical phonons accompanied by a dynamic dipole moment (IR-active phonons), the static dielectric constant must exceed $\varepsilon_{\infty}$. Only for materials without a dynamic dipole moment, such as crystalline $\mathrm{Si}, \varepsilon_{\text {st }}$ equals $\varepsilon_{\infty}$. As crystalline IV-VI semiconductors such as GeTe are characterized by very high values of the Born effective charge $[7,34]$, it is very difficult to imagine that for the corresponding amorphous material a vanishing Born effective charge, i.e., no dipole moment, is found. Indeed, DFT calculations revealed a nonvanishing Born effective charge for amorphous GeTe [35] and optical phonon modes have been observed by $\mathrm{THz}$ spectroscopy [36]. These findings are clearly incompatible with the statement that $\varepsilon_{\text {st }}$ equals $\varepsilon_{\infty}$ and call for a thorough investigation via impedance spectroscopy data.

It is possible that previous impedance spectroscopy data have suffered from shortcomings. Prokhorov et al. [31] and $\mathrm{Li}$ et al. [37] reported that the resistivity of $\mathrm{Ge}_{2} \mathrm{Sb}_{2} \mathrm{Te}_{5}$ decreases by merely 1 order of magnitude on crystallization. This observation is in conflict with the majority of studies agreeing on a resistivity decrease of about 3-4 orders of magnitude $[9,38]$ when crystallizing PCMs. This discrepancy might be a hint that the device resistance actually originates from highly resistive interface layers between the electrodes and the phasechange material. Indeed, it was R. A. Street et al. [39] who already emphasized the detrimental impact of contact-related effects in measurements on $\mathrm{As}_{2} \mathrm{Se}_{3}$, a composition closely related to PCMs. We therefore put special emphasis on the verification that our data are not affected by interface effects.

There are two additional aspects where our investigation goes beyond the scope of previous investigations. While previous investigations have studied the dielectric response either in the radio-frequency range or in the $\mathrm{THz}$ range, we investigated the entire frequency response from the DC limit to the near-infrared range. Furthermore, by comparing measurement results of different compounds, we were also able to study stoichiometry trends.

In this work, impedance spectroscopy measurements using a vector network analyzer covering the frequency range between $9 \mathrm{kHz}$ and $3 \mathrm{GHz}$ were performed to study the low-frequency dielectric constant and the AC conductivity of amorphous PCMs. To search for stoichiometric trends, we have investigated a number of compositions on the pseudobinary line between $\mathrm{GeTe}$ and $\mathrm{Sb}_{2} \mathrm{Te}_{3}$, focusing on the region between GeTe and $\mathrm{Ge}_{1} \mathrm{Sb}_{2} \mathrm{Te}_{4}$. The impedance spectroscopy experiments are corroborated and complemented by FTIR measurements investigating the full infrared range.

\section{EXPERIMENTAL DETAILS}

\section{A. Impedance spectroscopy}

As the endeavor of performing impedance spectroscopy experiments on PCMs is by no means trivial, we would like to start by explaining some basic considerations as well as some pitfalls with respect to the sample preparation and measurement process. Figures 1(a) and 1(b) illustrate the two contact geometries which can, in principle, be used for electrical measurements on thin films. It can be readily seen that the two geometries differ significantly with respect to the distribution of electrical field lines. In the in-plane geometry [Fig. 1(a)], only an insignificant portion of the field lines run through the phase-change material. The dielectric response being dominated by a mixture of the dielectric constants of substrate and air will be essentially insensitive to the dielectric constant of the phase-change layer. However, as the current is confined to the phase-change film in both cases, both geometries, in-plane and sandwich, can be employed to study the real part of the AC conductivity $\sigma_{1}(\omega)$. Consequently, experiments utilizing the in-plane geometry, such as [31,32], are suitable for deriving $\sigma_{1}(\omega)$, but they must not be employed (a)

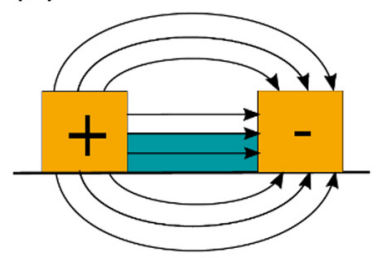

(c)

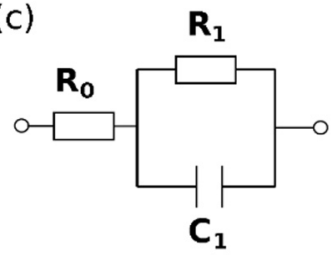

(b)
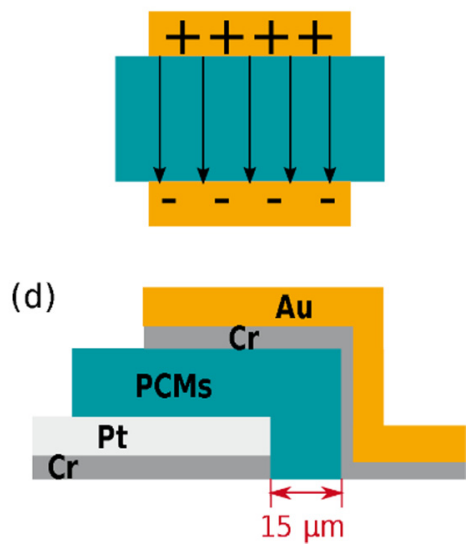

FIG. 1. Electric field distribution in in-plane structures (a) and in sandwich structures (b). Equivalent circuit for sandwich structures (c). $C_{1}$ and $R_{1}$ refer to the capacitance and the resistance of the PCM layer shown in (d), whereas $R_{0}$ denotes the contact resistance. (d) Cross-sectional structure of the impedance spectroscopy samples. 
for obtaining the real part of the dielectric function $\varepsilon_{1}(\omega)$. Therefore, the sandwich geometry [Fig. 1(b)], where a thin layer of amorphous PCM is inserted between two metal electrodes, is employed for all electrical measurements in this study.

The complex impedance $Z$ of the plate-capacitor-like structure can be understood as a parallel circuit composed of an Ohmic resistor $R_{1}=d /(A \sigma)$, representing the real part of the $\mathrm{AC}$ conductivity and a capacitor $C_{1}=\left(\varepsilon_{0} \varepsilon_{1} A\right) / d$, representing the real part of the dielectric function $\varepsilon_{1}$, as shown in Fig. 1(c), where $A$ is the cross-sectional area and $d$ is the thickness of the film. At frequencies above the transition frequency,

$$
f_{\mathrm{RC}}=\left(2 \pi R_{1} C_{1}\right)^{-1}=\frac{\sigma}{2 \pi \varepsilon_{0} \varepsilon_{1}},
$$

which is defined by $R_{1} \equiv\left|Z_{\mathrm{C}}\right|:=\left(\omega C_{1}\right)^{-1}$, the capacitor $C_{1}$ becomes dominant, and $\varepsilon_{1}$ can be determined. Note that $f_{\mathrm{RC}}$ is independent of the aspect ratio and depends only on the conductivity and the dielectric function of the material under investigation. With room-temperature conductivities of up to $10^{-3} \mathrm{~S} / \mathrm{cm}$, transition frequencies of a few $10-100 \mathrm{MHz}$ can be expected for amorphous PCMs. Obviously, a system covering a broad frequency range extending into the $\mathrm{GHz}$ range is desirable.

To this end, we employed a Rohde \& Schwarz ZVL3 vector network analyzer (VNA). Using two high-frequency coaxial cables and two contact head $Z$ probes (CascadeMicrotech, model Z 040 K3N GST 150), the two electrodes of the capacitorlike sandwich structures are connected to the two ports of the VNA. This way, the reflection scattering parameter $S_{11}$ as well as the transmission scattering parameter $S_{21}$ can be obtained between $9 \mathrm{kHz}$ and $3 \mathrm{GHz}$. The complex impedance of the sample $Z_{D U T}$ can be deduced from the scattering parameters by

$$
\begin{gathered}
Z_{D U T}=\frac{2 Z_{0} S_{11}}{1-S_{11}}, \\
Z_{D U T}=\frac{2 Z_{0}\left(1-S_{21}\right)}{S_{21}},
\end{gathered}
$$

where $Z_{0} \equiv 50 \Omega$ is the characteristic wave impedance of the VNA, the coaxial cables, and the contact heads. We probed all devices in reflection $\left(S_{11}\right)$ and transmission $\left(S_{21}\right)$. As is exemplarily shown in the Supplemental Material [40], the impedances obtained from $S_{11}$ and $S_{21}$ are in good agreement. However, as the transmission signal is less noisy, we will focus on the results obtained from Eq. (3) in the following.

Proper calibration up to $\mathrm{GHz}$ frequencies is mandatory because the wavelength is shorter than the cables at $\mathrm{GHz}$ frequencies. We performed a TOSM calibration (thru, open, short, match) at the tips of the contact heads using the calibration substrate provided by the manufacturer of the contact heads. However, the touch-down points of the two contact heads are separated by $600 \mu \mathrm{m}$ with the much smaller capacitorlike sample structure in the middle. As a consequence, the finite conductivity of the electrode material accounts for a small contact resistance $R_{0}$ of a few ohms, which is also included in the equivalent circuit in Fig. 1(c).
Thus, the complex impedance can be expressed by

$$
Z_{D U T}=R_{0}+\frac{R_{1}}{1+i\left(\omega R_{1} C_{1}\right)},
$$

where the AC conductivity and the dielectric permittivity can derived from

$$
\begin{gathered}
\sigma_{A C}=\frac{d}{A} \operatorname{Re}\left(\frac{1}{Z_{D U T}-R_{0}}\right), \\
\varepsilon_{1}=\frac{d}{A} \operatorname{Im}\left(\frac{1}{Z_{D U T}-R_{0}}\right)\left(\frac{1}{2 \pi f \varepsilon_{0}}\right) .
\end{gathered}
$$

As can be seen from solving Eqs. (2) and (3) for $S_{11}$ and $S_{21}$, the method is most sensitive if the device impedance $Z_{D U T}$ is close to the characteristic impedance $Z_{0} \equiv 50 \Omega$. With the film thickness limited to a few hundred nanometers for practical reasons, the area of the capacitorlike sandwich structures has to be tuned to meet this condition. To this end, we resorted to an optical lithography process, which facilitates varying the capacitor size between $10 \times 10 \mu \mathrm{m}^{2}$ and $400 \times 400 \mu \mathrm{m}^{2}$. Figure 1(d) depicts a sketch of the lithographically patterned layer stack stating typical layer thicknesses. The structures were built on polished $20 \times 20 \times 0.53 \mathrm{~mm}^{3}$-sized sapphire substrates. The metal electrodes (100 nm Au/5 nm Cr and $100 \mathrm{~nm} \mathrm{Pt} / 5 \mathrm{~nm} \mathrm{Cr}$ ) were grown by evaporation. We employed platinum instead of gold as the bottom electrode because gold might diffuse upon heating during the lithography process (up to $115^{\circ} \mathrm{C}$ ). However, gold can be used as a top electrode since, at its stage of installation during device formation, heating is no longer required. Amorphous PCMs were deposited from stoichiometric targets of $99.99 \%$ purity by DC magnetron sputtering at base pressures of $\leqslant 2 \times 10^{-6}$ mbar with $20 \mathrm{sccm}$ argon flow and constant power of $20 \mathrm{~W}$ in the sputtering system LS 320S (manufacturer: Pfeiffer/Von Ardenne). The argon pressure during the sputtering process was $3.4 \times 10^{-3} \mathrm{mbar}$, and the distance between the target and substrate was $4 \mathrm{~cm}$.

\section{B. FTIR spectroscopy}

The above-mentioned sputter tool was also employed to fabricate samples for the optical measurements. Amorphous phase-change layers of 800-1000 nm thickness were deposited on double-side-polished, highly resistive $(>5000 \mathrm{k} \Omega \mathrm{cm})$ silicon (100) wafers. Far-infrared to near-infrared spectra $\left(20-12000 \mathrm{~cm}^{-1}\right.$, i.e., $\left.0.6 \mathrm{THz}-360 \mathrm{THz}\right)$ were recorded by a Bruker IFS 66/v FTIR spectrometer in transmission geometry. The spectra were subsequently analyzed by approximating the dielectric function by a regular array of oscillatory terms. Details of this procedure can be found elsewhere $[41,42]$. This Kramers-Kronig consistent approach yielded the complex dielectric function $\varepsilon(\omega)=\varepsilon_{1}(\omega)+i \varepsilon_{2}(\omega)$, and from this we got the conductivity $\sigma_{1}(\omega)=\varepsilon_{0} \omega \varepsilon_{2}(\omega)$.

\section{RESULTS AND DISCUSSION}

\section{A. Validity of our impedance spectroscopy measurements}

As was already mentioned in the Introduction, impedance spectroscopy data can be easily distorted by sample imperfections such as insulating interlayers (e.g., oxide layers), nonohmic contacts, or pinholes. Indeed, we revised the sample 


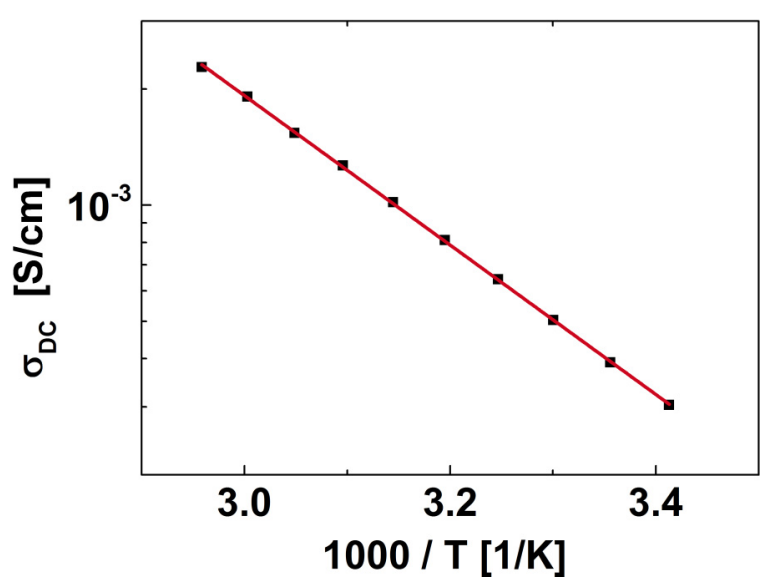

FIG. 2. Temperature dependence of the DC conductivity $\sigma_{\mathrm{DC}}$ derived from an amorphous $\mathrm{Ge}_{3} \mathrm{Sb}_{2} \mathrm{Te}_{6}$ sandwich structure. The activation energy $E_{A}$ and the preexponential factor $\sigma_{0}$ according to Eq. (7) are $0.38 \mathrm{eV}$ and $\sim 1236 \mathrm{~S} / \mathrm{cm}$, respectively.

preparation to eliminate the formation of highly resistive interface layers. An example of a measurement where insulating layers resulting from faulty sample preparation affect the impedance spectroscopy results can be found in the Supplemental Material [40]. The presence of interlayers, broken contacts, or pinholes adds extra elements to the equivalent circuit shown in Fig. 1(c) and, thus, renders Eqs. (5) and (6) invalid. As a consequence, erroneous results for $\varepsilon(\omega)$ and $\sigma(\omega)$ are obtained. The following validation experiments, however, demonstrate that the revision of our sample preparation process was successful, i.e., that our data are not affected by such shortcomings.

In a first step, we probed the DC conductivity of the sandwich structures using a source measure unit (Keithley SCS 4200) and a temperature-controlled probe station. Figure 2 depicts the DC conductivity and its temperature dependence for a $\mathrm{Ge}_{3} \mathrm{Sb}_{2} \mathrm{Te}_{6}$ sandwich structure. The data nicely follow the Arrhenius law

$$
\sigma(T)=\sigma_{0} e^{-\frac{E_{A}}{k_{B} T}}
$$

where the activation energy $E_{A}$ and the prefactor $\sigma_{0}$ are $0.38 \mathrm{eV}$ and $1236 \mathrm{~S} / \mathrm{cm}$, respectively. The room-temperature conductivity is $\sigma_{20}{ }^{\circ} \mathrm{C}=3 \times 10^{-4} \mathrm{~S} / \mathrm{cm}$. From in-plane samples, we obtained $E_{A}=0.38 \mathrm{eV}$ and, depending on the thermal history of the film (resistance drift), $\sigma_{20^{\circ} \mathrm{C}}=2-4 \times 10^{-4} \mathrm{~S} / \mathrm{cm}$. This excellent agreement between the sandwich structures and the in-plane samples is very comforting. Owing to the aspect ratio, in-plane samples are less prone to be compromised by interface effects or contact effects. And due to the lateral current flow, also pinholes should not matter. The fact that the electrical properties obtained from both geometries are in line is, therefore, strong evidence that the sandwich structures are not affected by these shortcomings.

In addition, we have also verified the scaling behavior of the device impedance with film thickness and capacitor area. As can be seen from Fig. 3, the impedance scales properly with film thickness and device area, i.e. $\epsilon$ does not depend on the particular thickness and area of the sandwich device. The small variation discernible in Fig. 3 amounts to 3\%. The geometry

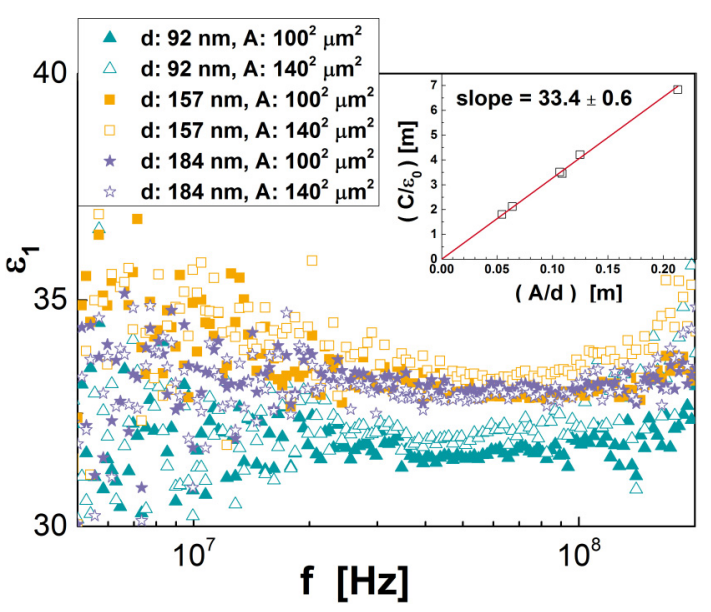

FIG. 3. Dielectric permittivity $\varepsilon_{1}$ as a function of frequency $f$ for amorphous $\mathrm{Ge}_{2} \mathrm{Sb}_{2} \mathrm{Te}_{5}$ obtained from different device geometries. Neither doubling the layer thickness $d$ nor doubling the capacitor area $A$ has a significant impact on the obtained permittivity. The inset graph displays $C / \varepsilon_{0}$ vs $A / d$. As expected, the data points obtained from different geometries are located on a straight line through the origin, where the slope of 33.4 corresponds to $\varepsilon_{1}$.

error of the lithography process can readily account for this variation. As is demonstrated in Sec. 3 of the Supplemental Material [40], also the device resistance scales properly with thickness and area. However, owing to the already mentioned resistance drift, the error bars are slightly larger in this case. If the data were distorted by interface effects or contact problems, the results should depend on the film thickness. Therefore, the good agreement between data obtained from phase-change layers of different thicknesses again indicates the absence of interface and contact problems.

Hence, we have confirmed that the DC resistivity as well as its temperature dependence is perfectly in line with data obtained independently from in-plane measurements. Furthermore, the device properties scale as expected with film thickness and device area. This indicates that our measurements are not affected by interface effects, non-Ohmic contacts, or pinholes.

\section{B. Frequency-dependent permittivity and conductivity}

Figure 4 depicts a Nyquist plot $\left(-Z_{2}\right.$ vs $\left.Z_{1}\right)$ of the impedance spectroscopy data derived by Eq. (3) from $S_{21}$. Apparently, the data points are located on semicircles. As it is well known that a single RC element produces a semicircle in a Nyquist plot [43]; this behavior is expected from Eq. (4) and Fig. 1(c).

Following the equivalent circuit described by Fig. 1(c) and Eq. (4), three cases are identified in terms of frequency regimes. In the DC limit, the impedance is dominated by the resistance of the phase-change material layer $R_{1}$ and is, therefore, purely Ohmic. If a contact resistance $R_{0}$ (in our case about $6 \Omega$ ) is taken into account, the DC limit reads $Z_{\mathrm{st}}=R_{0}+R_{1}$. This corresponds to the rightmost point of the semicircle in Fig. 4. With increasing frequency, the transition to capacitive behavior sets in as the capacitor $C_{1}$ becomes conductive. The transition frequency $f_{\mathrm{RC}}$ [see 


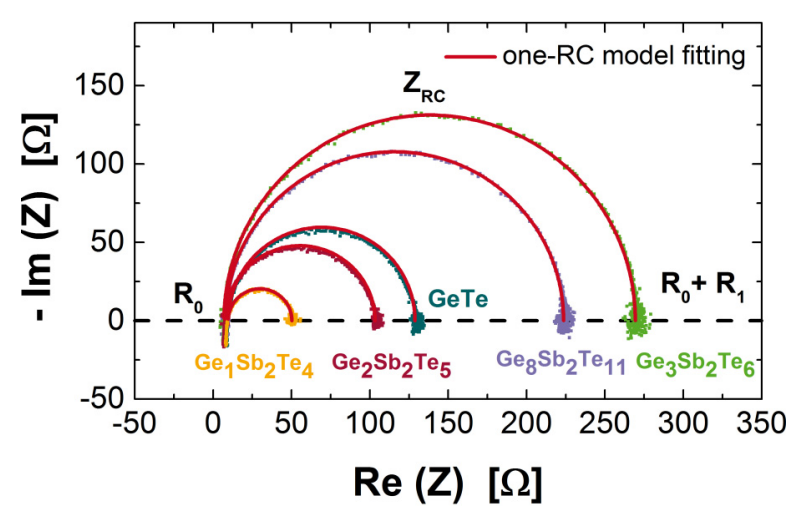

FIG. 4. The Nyquist plot displays the impedance spectroscopy

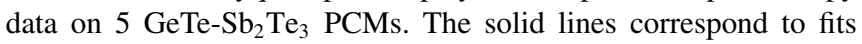
according to the one-RC model defined by Fig. 1(c) and Eq. (4).

Eq. (1)] corresponds to the topmost point of the semicircle: $Z_{\mathrm{RC}}=R_{0}+(1-i) R_{1} / 2$. Here, the equal contributions of $R_{1}$ and $C_{1}$ to the current flow result in a phase angle of $-\pi / 4$. In the high-frequency limit, the contact resistor $R_{0}$ dominates and the behavior becomes Ohmic again: $Z_{\infty}=R_{0}$.

The red lines, representing fits to Eq. (4), indicate that the very simple model already offers a very accurate description of the data. The finding of the Nyquist diagram displaying only one semicircle is in striking contrast to literature data, where two semicircles are observed and consequently models comprised of two RC elements are invoked to interpret the data [31,37]. The additional RC element is typically attributed to highly resistive contacts [44] or to highly resistive grain boundaries $[31,45,46]$, where the latter, of course, only makes sense in crystalline systems. As the explanations for the additional RC element are somewhat awkward, it is comforting that our data reveal just one RC element.

The agreement with the one-RC model in Fig. 4 already suggests that $\sigma(\omega)$ is fairly frequency independent. In Figs. 5 and 6 , we explicitly check the frequency dependence of $\sigma(\omega)$ and $\varepsilon(\omega)$ by employing Eqs. (5) and (6) to derive both quantities directly from the experimental data. Figure 5 demonstrates that $\sigma(\omega)$ is frequency independent in the entire

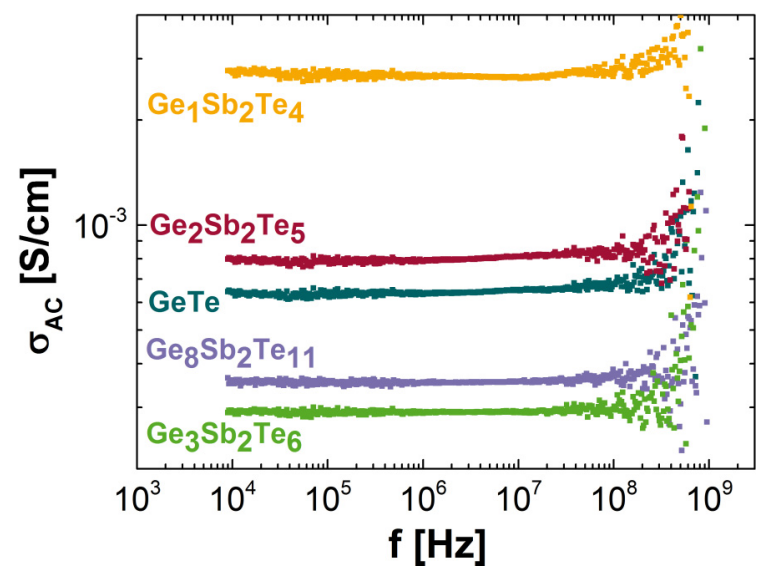

FIG. 5. AC conductivity $\sigma_{\mathrm{AC}}$ of amorphous PCMs. In the entire frequency range, no frequency dependence is observed. This finding is in line with transport by extended states at the mobility edge.

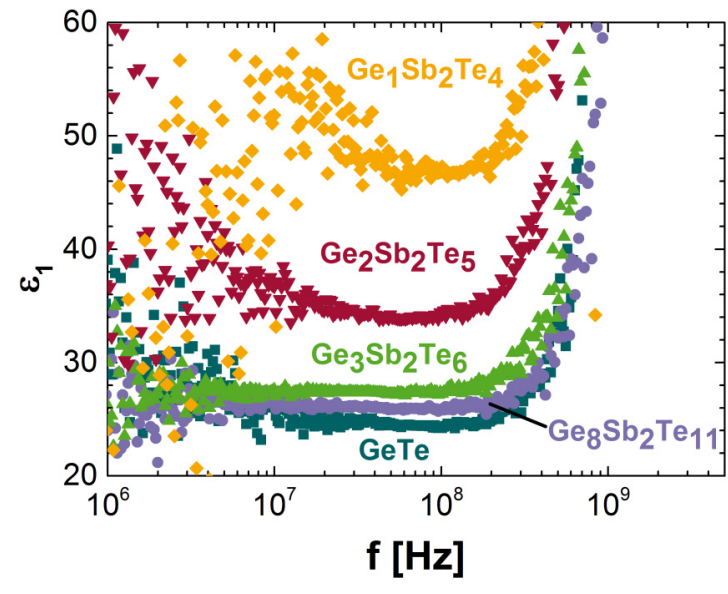

FIG. 6. Dielectric permittivity $\varepsilon_{1}$ of five amorphous $\mathrm{GeTe}-\mathrm{Sb}_{2} \mathrm{Te}_{3}$ alloys. Section III B explains the origin of the noise at low frequencies and the upturn at high frequencies. In the intermediate range, where reliable data could be acquired, no frequency dependence is discernible.

measurement range. At frequencies above $100 \mathrm{MHz}$, the capacitance $C_{1}$ dominates, making the determination of $R_{1}$ and, thus, $\sigma(\omega)$ increasingly noisy. The absence of a frequency dependence is by no means trivial, since this finding differs from literature on other chalcogenides such as $\mathrm{As}_{2} \mathrm{Te}_{3}$ [47]. If a frequency-dependent conductivity (often of the type $\sigma \propto \omega^{\mathrm{s}}$ ) is observed, this phenomenon is usually attributed to hopping transport [48]. The "flat" conductivity spectra we obtained are perfectly in line with charge transport in extended states at the mobility edge ("band transport"). Given the comparably high conductivity of amorphous PCMs, this picture seems plausible.

Figure 6 presents the dielectric permittivity as derived from Eq. (6). Far below the transition frequency, the total impedance $Z_{D U T}$ is dominated by the conductivity of the film $\left(\rightarrow R_{1}\right)$ and, hence, no determination of the permittivity $\left(C_{1}\right)$ is possible. At frequencies close to $3 \mathrm{GHz}, Z_{D U T}$ is governed by the contact resistance $R_{0}$. Small errors in the determination of $R_{0}$, therefore, have a large impact on $\varepsilon(\omega)$ in this region. In addition, miniscule length variations between the waveguide structure employed for calibration and the actual samples can give rise to phase errors in this region. The upturn of $\varepsilon(\omega)$, therefore, does not reflect the actual frequency dependence. In the frequency range, where reliable data could be obtained, the dielectric permittivity is constant within the margin of error. This result is again not trivial. Literature data on other amorphous materials frequently display changes in the permittivity with frequency, which are then explained in terms of dielectric relaxations [48].

\section{Impact of IR-active phonons on the static dielectric constant}

Table I and Fig. 7 compile the conductivities and static dielectric constants obtained in the previous section by impedance spectroscopy. A comparison between the static dielectric constant $\varepsilon_{\mathrm{st}}$ derived by impedance spectroscopy and the optical dielectric constant $\varepsilon_{\infty}$ obtained by midinfrared FTIR is tempting. As discussed in the Introduction, 
TABLE I. Summary of the impedance spectroscopy and FTIR results. The DC conductivities $\sigma_{\mathrm{DC}}$ at room temperature (i.e., $20^{\circ} \mathrm{C}$ ) from in-plane samples and sandwich structures, the static dielectric constants $\varepsilon_{\mathrm{st}}$ from impedance spectroscopy (IS) and FTIR, the optical dielectric constants $\varepsilon_{\infty}$ from FTIR, and the spectral weights of phonons (SWP) are listed.

\begin{tabular}{|c|c|c|c|c|c|c|}
\hline Quantities Unit Methods & $\sigma_{\mathrm{DC}} \mathrm{S} / \mathrm{cm}$ In-plane & $\sigma_{\mathrm{DC}} \mathrm{S} / \mathrm{cm} \mathrm{IS}$ & $\varepsilon_{\mathrm{st}} \mathrm{IS}$ & $\varepsilon_{\mathrm{st}}$ FTIR & $\varepsilon_{\infty}$ FTIR & SWP S $/ \mathrm{cm}^{2}$ FTIR \\
\hline $\mathrm{GeTe}$ & $(8 \pm 2) \times 10^{-4}$ & $6.2 \times 10^{-4}$ & 24.4 & 22.5 & 13.2 & 2018 \\
\hline $\mathrm{Ge}_{8} \mathrm{Sb}_{2} \mathrm{Te}_{11}$ & $(6 \pm 1.5) \times 10^{-4}$ & $3.6 \times 10^{-4}$ & 25.8 & 23.1 & 13.3 & 1876 \\
\hline $\mathrm{Ge}_{3} \mathrm{Sb}_{2} \mathrm{Te}_{6}$ & $3.6 \times 10^{-4}$ & $3.4 \times 10^{-4}$ & 27.4 & 25.5 & $14.7^{\mathrm{a}}$ & 2059 \\
\hline $\mathrm{Ge}_{2} \mathrm{Sb}_{2} \mathrm{Te}_{5}$ & $(11 \pm 3) \times 10^{-4}$ & $7.7 \times 10^{-4}$ & 34.0 & 33.3 & $16^{\mathrm{a}}$ & 2242 \\
\hline $\mathrm{Ge}_{1} \mathrm{Sb}_{2} \mathrm{Te}_{4}$ & $(19 \pm 5) \times 10^{-4}$ & $24.5 \times 10^{-4}$ & 47.0 & 52.0 & $16.6^{\mathrm{a}}$ & 4046 \\
\hline
\end{tabular}

${ }^{\text {a Reference } 8 .}$

$\varepsilon_{\mathrm{st}}>\varepsilon_{\infty}$ points to the presence of IR-active phonons. Indeed, for the pseudobinary GeTe- $\mathrm{Sb}_{2} \mathrm{Te}_{3}$ systems, the impedancespectroscopy-based values of $\varepsilon_{\mathrm{st}}$ ranging from 24 to 47 clearly exceed the FTIR-based values of $\varepsilon_{\infty}$, which are always close to 15 in these compositions [8]. Thus, in contrast to literature data, where $\varepsilon_{\mathrm{st}} \approx 16$ was reported for $\mathrm{Ge}_{2} \mathrm{Sb}_{2} \mathrm{Te}_{5}$ [31], we find evidence of significant IR-active phonons in all pseudobinary PCMs.

Therefore, we extended the midinfrared FTIR analysis already performed by Shportko et al. [8] to the far-infrared range (down to $20 \mathrm{~cm}^{-1}$ ). These measurements allow for identifying the IR-active phonons and can, thus, shed more light on the origin of the stoichiometry trend in the pseudobinary GeTe-Sb $\mathrm{Te}_{3}$ system. The far-infrared transmission spectra are depicted in the Supplemental Material [40]. A transformation consistent with the Kramers-Kronig relations yields the dielectric functions displayed in Fig. 8. Indeed, the pronounced absorption features discernible in the imaginary parts $\varepsilon_{2}(\omega)$ below $250 \mathrm{~cm}^{-1}$ can unequivocally be attributed to IR-active phonons.

A closer inspection with respect to the stoichiometry trend leads to the following three observations: First, the absorption spectrum of GeTe appears to be better defined (sharper) than those of the $\mathrm{Sb}_{2} \mathrm{Te}_{3}$-containing compositions. The reason for this effect may be that $\mathrm{GeTe}$ - as a truly binary alloy-features less disorder than the ternary compounds. Second, on increasing the $\mathrm{Sb}_{2} \mathrm{Te}_{3}$ content, the modes clearly

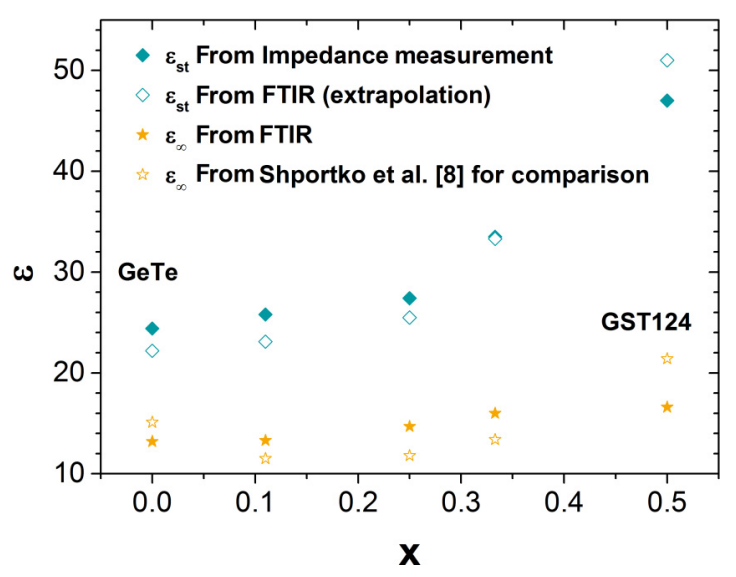

FIG. 7. Static $\left(\varepsilon_{\mathrm{st}}\right)$ and optical $\left(\varepsilon_{\infty}\right)$ dielectric constants of amorphous $(\mathrm{GeTe})_{(1-x)}-\left(\mathrm{Sb}_{2} \mathrm{Te}_{3}\right)_{x}$ alloys from impedance spectroscopy and FTIR. While $\varepsilon_{\infty}$ depends only weakly on the stoichiometry, there is a marked surge in $\varepsilon_{\mathrm{st}}$ on increasing the $\mathrm{Sb}_{2} \mathrm{Te}_{3}$ content. shift to lower frequencies. This effect can be partly attributed to the increase of the reduced mass for Sb-Te vibrations as compared to GeTe vibrations. Yet, it is very likely that an additional contribution comes from a weaker bonding in the Sb-rich compounds. Third, as can be seen from Table I, also the spectral weight of phonons increases continuously from GeTe to $\mathrm{Ge}_{1} \mathrm{Sb}_{2} \mathrm{Te}_{4}$. As we will explain in the following, this means that the increase in $\varepsilon_{\mathrm{st}}$ is not just a mere consequence of the phonon softening due to the larger mass of the material constituents.

The spectral weight of phonons can be obtained by integrating the optical conductivity $\sigma_{1}$ as

$$
\mathrm{SWP}=\int d \omega \sigma_{1}(\omega)=\frac{\pi}{2} \varepsilon_{0} \sum_{k} \Delta \varepsilon_{k} \Omega_{k}^{2},
$$

where all nonphononic contributions have been subtracted from $\sigma_{1}$. For phonons that can be described by harmonic Lorentz oscillators, this integral is equivalent to the expression at the right-hand side of Eq. (8). There, $\Delta \varepsilon_{\mathrm{k}}$ is the phonon contribution to the dielectric constant and $\Omega_{\mathrm{k}}$ is its resonance frequency [41].

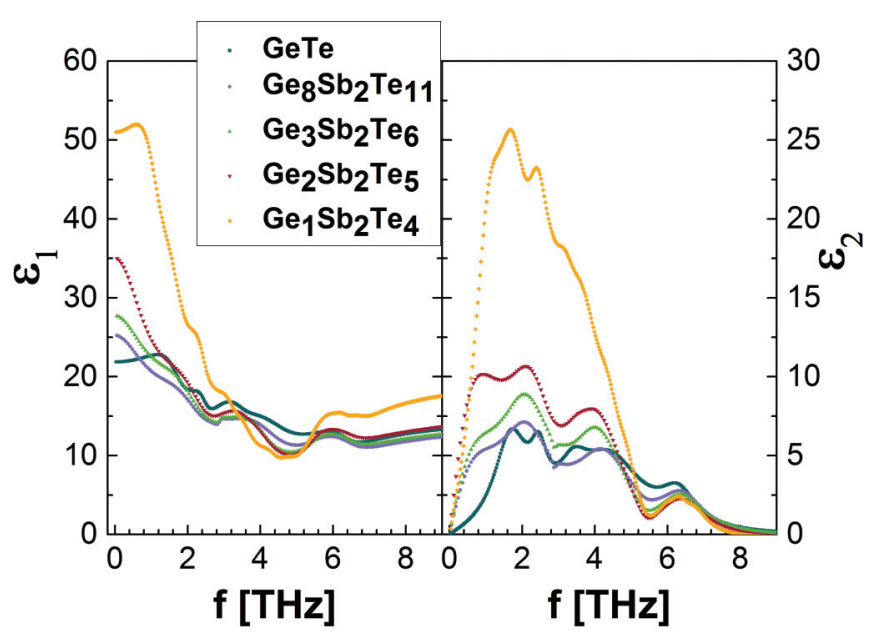

FIG. 8. Real and imaginary parts of the dielectric function obtained from the transmission spectra depicted in the Supplemental Material [40]. Pronounced phonon absorption is discernible below $9 \mathrm{THz}\left(300 \mathrm{~cm}^{-1}\right)$. Adding $\mathrm{Sb}_{2} \mathrm{Te}_{3}$ in the $\mathrm{GeTe}-\mathrm{Sb}_{2} \mathrm{Te}_{3}$ system induces a reduction of the phonon frequencies, probably a consequence of the Ge vs Sb mass difference. The extrapolation of the real part down to zero frequency reproduces the stoichiometry trend in $\varepsilon_{\mathrm{st}}$, which has already been observed by impedance spectroscopy in Fig. 6. 
Of course, conservation of phonon spectral weight implies that a reduction of the phonon frequencies induces an increase in the value of $\Delta \varepsilon=\varepsilon_{\mathrm{st}}-\varepsilon_{\infty}$, and hence of $\varepsilon_{\mathrm{st}}$. However, the fact that we observe a systematic increase in the spectral weight of phonons indicates that, in addition to the softening of the phonon frequencies, an enlargement of the dipole moment accounts for the larger values of $\varepsilon_{\mathrm{st}}$. Hence, on increasing the $\mathrm{Sb}$ content, also the Born effective charge gets larger.

The Born effective charge $Z_{k}$ can be obtained from the equations below:

$$
\begin{gathered}
\sum_{k} \frac{n_{k} Z_{k}^{2}}{m_{k}}=\frac{2 V}{\pi} \int \sigma_{1}(\omega) d \omega, \\
\sum_{k} n_{k} Z_{k}=0 .
\end{gathered}
$$

In Eq. (9) [49,50], we made an approximation that all $n_{k}$ atoms of the same species (mass $m_{k}$ ) have the same Born effective charge $Z_{k}$, where $V$ is the volume occupied by all atoms in the left-hand side summation. As can been seen in Eq. (10), the Born effective charge also respects charge neutrality. The determination of $Z_{k}$ is possible if we consider an isotropic material, so that the dielectric function is a scalar quantity, with just two different atoms, such as GeTe. For amorphous GeTe, a Born effective charge of around $+2(\mathrm{Ge})$ and -2 (Te), respectively, is obtained. This number is in good agreement with DFT calculations, which determine the Born effective charge to values of approximately 2 [35]. Interesting enough, crystalline GeTe has been calculated to have an average Born effective charge of 4.6 [7,51], which depends strongly on atomic arrangement. Hence crystallization leads to a significant increase in the Born effective charge. Since the Born effective charge, as already mentioned above, is a bond indicator, this implies that crystallization of GeTe, as well as the other PCMs studied here, has a pronounced effect on the bonding mechanism utilized in this material. We are not aware of any other material class which shows such a pronounced change of bonding upon crystallization. This phenomenon has recently also been confirmed for $\mathrm{Ge}_{2} \mathrm{Sb}_{2} \mathrm{Te}_{5}$ [52], where the increase in the Born effective charge upon crystallization is explained by an ionic bonding contribution. Thus, the increase of the Born effective charge upon crystallization seems to be a generic property of PCMs. Yet, explaining this finding by an ionic bond contribution is not obvious [52], given the fact that in GeTe the difference in electronegativity is very small, since Ge (2.01) and Te (2.1) [53] have almost identical values. This is quite different from the scenario encountered in clearly ionic compounds such as $\mathrm{PbTiO}_{3}$ ( $\mathrm{Pb} 2.33$, Ti 1.54, O 3.44) [53].

Given the atomic masses of $\mathrm{Ge}, \mathrm{Sb}$, and $\mathrm{Te}$, it is hard to imagine that phonons featuring frequencies below the lower limit of our measurement range $\left(20 \mathrm{~cm}^{-1}\right)$ exist. Thus, employing the FTIR data to extrapolate the dielectric function down to zero frequency is worthwhile. As can be seen from Fig. 7 and Table I, the values of $\varepsilon_{\mathrm{st}}$ derived from the far-infrared FTIR data are in excellent agreement with those obtained from impedance spectroscopy for all stoichiometries studied. If one of the two methods (FTIR and impedance spectroscopy) had been compromised by shortcomings, it would be highly unlikely that both techniques independently produce the same results. Thus, the good agreement substantiates the validity of both methods and corroborates the observation that, in contradiction to literature data, $\varepsilon_{\mathrm{st}}$ clearly exceeds $\varepsilon_{\infty}$. Moreover, it indicates that the FTIR frequency range was sufficiently broad to cover all major optical excitations below the interband transition, i.e., as we expected, there are no significant optical excitations between the lowest FTIR frequency $\left(20 \mathrm{~cm}^{-1}\right)$ and the DC limit.

\section{CONCLUSIONS}

We carried out impedance spectroscopy and far-infrared FTIR measurements on thin films of amorphous PCMs. As the sandwich structures required for impedance spectroscopy measurements are prone to be affected by interface layers and as our results contradict with existing literature data, we performed reference experiments explicitly demonstrating the absence of such interface effects and other shortcomings in our samples.

Frequency dependences of the AC conductivity $\sigma(\omega)$ and the AC permittivity $\varepsilon(\omega)$ in impedance spectroscopy experiments are usually explained in terms of dielectric relaxations and hopping transport, respectively. Our impedance spectroscopy data can be readily understood in terms of a single RC element comprised of a frequency-independent resistor and a frequency-independent capacitor, i.e., both $\varepsilon(\omega)$ and $\sigma(\omega)$, do not display any frequency dependence in the impedance spectroscopy range ( $\mathrm{kHz}$ to $\mathrm{GHz}$ frequencies). No evidence of relaxations or hopping transport was observed. Given the comparably high room-temperature conductivity of amorphous PCMs, the conjecture of transport by extended states at the mobility edge appears plausible.

In striking contrast to the existing literature, the static dielectric constant $\varepsilon_{\text {st }}$ clearly exceeds the optical dielectric constant $\varepsilon_{\infty}$ in the $\mathrm{GeTe}-\mathrm{Sb}_{2} \mathrm{Te}_{3}$ system, where $\varepsilon_{\mathrm{st}}$ increases continuously with increasing $\mathrm{Sb}_{2} \mathrm{Te}_{3}$ content. This study reports on the difference between $\varepsilon_{\mathrm{st}}$ and $\varepsilon_{\infty}$ and the stoichiometry trend in the pseudobinary system. We expect that these data on the static dielectric constant will be of great help in modeling capacitive effects in PRAM device simulations or in gauging DFT structure models.

Our findings are backed up by our far-infrared FTIR measurements, which independently corroborate the values of the static dielectric constant and unambiguously attribute the difference between the static dielectric constant and the optical dielectric constant to IR-active phonons in the region between 20 and $250 \mathrm{~cm}^{-1}$. The good agreement between the static dielectric constant obtained from impedance spectroscopy and the FTIR-based extrapolation of the static dielectric constant indicates that all significant optical excitations could be detected by far-infrared FTIR and that we have obtained the complete dielectric function of amorphous PCMs between the DC limit and the first interband transition. The FTIR spectra reveal a shift to lower phonon frequencies on increasing the $\mathrm{Sb}_{2} \mathrm{Te}_{3}$ content. In addition, the increasing spectral weight of phonons indicates that the strength of the phonons is enlarged at the same time. As was already noticed by Shportko et al. [8], the presence of resonant bonding in crystalline PCMs leads to a doubling of $\varepsilon_{\infty}$ on crystallization. Here further experimental evidence is presented that both the Born effective charge and the static dielectric constant also change significantly upon 
crystallization. This should help to unravel the changes further, which characterizes the change in bonding mechanism that accompanies crystallization in PCMs.

\section{ACKNOWLEDGMENTS}

The authors are grateful for support from the China Scholarship Council (CSC) and the Deutsche Forschungsge- meinschaft (SFB917). Moreover, the research leading to these results received funding from the European Union Seventh Framework Programme (FP7/2007-2013) under Grant Agreement No. 340698. The authors also acknowledge access to the cleanroom of the Helmholtz Nanoelectronic Facility (HNF) of Forschungszentrum Jülich, and insightful discussions with Peter Zalden, Wei Zhang, and Konstantin Shportko.
[1] S. R. Ovshinsky, Phys. Rev. Lett. 21, 1450 (1968).

[2] M. Wuttig and N. Yamada, Nat. Mater. 6, 824 (2007).

[3] G. W. Burr, M. J. Breitwisch, M. Franceschini, D. Garetto, K. Gopalakrishnan, B. Jackson, B. Kurdi, C. Lam, L. A. Lastras, A. Padilla, B. Rajendran, S. Raoux, and R. S. Shenoy, J. Vac. Sci. Technol. B 28, 223 (2010).

[4] H. F. Hamann, M. O’Boyle, Y. C. Martin, M. Rooks, and H. K. Wickramasinghe, Nat. Mater. 5, 383 (2006).

[5] G. Bruns, P. Merkelbach, C. Schlockermann, M. Salinga, M. Wuttig, T. D. Happ, J. B. Philipp, and M. Kund, Appl. Phys. Lett. 95, 043108 (2009).

[6] C. S. Hwang, Adv. Electron. Mater. 1, 1400056 (2015).

[7] D. Lencer, M. Salinga, B. Grabowski, T. Hickel, J. Neugebauer, and M. Wuttig, Nat. Mater. 7, 972 (2008).

[8] K. Shportko, S. Kremers, M. Woda, D. Lencer, J. Robertson, and M. Wuttig, Nat. Mater. 7, 653 (2008).

[9] T. Siegrist, P. Jost, H. Volker, M. Woda, P. Merkelbach, C. Schlockermann, and M. Wuttig, Nat. Mater. 10, 202 (2011).

[10] W. Zhang, A. Thiess, P. Zalden, R. Zeller, P. H. Dederichs, J. Y. Raty, M. Wuttig, S. Blugel, and R. Mazzarello, Nat. Mater. 11, 952 (2012).

[11] B. Huang and J. Robertson, Phys. Rev. B 81, 081204 (2010).

[12] S. Gabardi, S. Caravati, G. C. Sosso, J. Behler, and M. Bernasconi, Phys. Rev. B 92, 054201 (2015).

[13] W. H. Zachariasen, J. Am. Chem. Soc. 54, 3841 (1932).

[14] A. V. Kolobov, P. Fons, A. I. Frenkel, A. L. Ankudinov, J. Tominaga, and T. Uruga, Nat. Mater. 3, 703 (2004).

[15] P. Jóvári, I. Kaban, J. Steiner, B. Beuneu, A. Schöps, and M. A. Webb, Phys. Rev. B 77, 035202 (2008).

[16] M. Krbal, A. V. Kolobov, P. Fons, J. Tominaga, S. R. Elliott, J. Hegedus, and T. Uruga, Phys. Rev. B 83, 054203 (2011).

[17] E. Matsubara, S. Okada, T. Ichitsubo, T. Kawaguchi, A. Hirata, P. F. Guan, K. Tokuda, K. Tanimura, T. Matsunaga, M. W. Chen, and N. Yamada, Phys. Rev. Lett. 117, 135501 (2016).

[18] V. L. Deringer, W. Zhang, M. Lumeij, S. Maintz, M. Wuttig, R. Mazzarello, and R. Dronskowski, Angew. Chem. Int. Ed. 53, 10817 (2014).

[19] W. Wełnic and M. Wuttig, Mater. Today 11, 20 (2008).

[20] D. Krebs, T. Bachmann, P. Jonnalagadda, L. Dellmann, and S. Raoux, New J. Phys. 16, 043015 (2014).

[21] J. Akola and R. O. Jones, Phys. Rev. B 76, 235201 (2007).

[22] J. Akola, R. O. Jones, S. Kohara, S. Kimura, K. Kobayashi, M. Takata, T. Matsunaga, R. Kojima, and N. Yamada, Phys. Rev. B 80, 020201 (2009).

[23] J. Hegedus and S. R. Elliott, Nat. Mater. 7, 399 (2008).

[24] S. Caravati, M. Bernasconi, T. D. Kühne, M. Krack, and M. Parrinello, Phys. Rev. Lett. 102, 205502 (2009).

[25] S. Raoux, G. W. Burr, M. J. Breitwisch, C. T. Rettner, Y. C. Chen, R. M. Shelby, M. Salinga, D. Krebs, S. H. Chen,
H. L. Lung, and C. H. Lam, IBM J. Res. Dev. 52, 465 (2008).

[26] D. Lencer, M. Salinga, and M. Wuttig, Adv. Mater. 23, 2030 (2011).

[27] S. Jakobs, A. von Hoegen et al., Resonance Bonding in Crystalline Solids: How Can It Be Broken? (unpublished, contact "M. Wuttig" for details).

[28] D. Emin, in Linear and Nonlinear Electron Transport in Solids, edited by J. T. Devreese and V. E. V. Doren (Springer, New York, 1976), Vol. 17, p. 409.

[29] D. Ielmini, D. Sharma, S. Lavizzari, and A. L. Lacaita, IEEE Trans. Electron Dev. 56, 1070 (2009).

[30] A. S. Maan, D. R. Goyal, and A. Kumar, Rev. Phys. Appl. 24, 1071 (1989).

[31] E. Prokhorov, J. J. Gervacio-Arciniega, G. Luna-Bárcenas, Y. Kovalenko, F. J. Espinoza-Beltrán, and G. Trápaga, J. Appl. Phys. 113, 113705 (2013).

[32] R. R. Santos, E. Prokhorov, J. G. Hernández, G. L. Bárcenas, and Y. Kovalenko, J. Non-Cryst. Solids 356, 2541 (2010).

[33] Some literature refer to the static dielectric constant by $\varepsilon_{0}$, which is also the standard symbol used for the vacuum permittivity $\left(8.85 \times 10^{-12} \mathrm{~F} / \mathrm{m}\right)$. Here we resort to the following notation: vacuum permittivity $\left(\varepsilon_{0}\right)$, static dielectric constant $\left(\varepsilon_{\mathrm{st}}\right)$, and optical dielectric constant $\left(\varepsilon_{\infty}\right)$.

[34] P. B. Littlewood, J. Phys. C: Solid State Phys. 12, 4459 (1979).

[35] J. Y. Raty, W. Zhang, J. Luckas, C. Chen, R. Mazzarello, C. Bichara, and M. Wuttig, Nat. Commun. 6, 7467 (2015).

[36] F. Kadlec, C. Kadlec, and P. Kuzel, Solid State Commun. 152, 852 (2012).

[37] Y. Li, Y. P. Zhong, J. J. Zhang, X. H. Xu, Q. Wang, L. Xu, H. J. Sun, and X. S. Miao, Appl. Phys. Lett. 103, 043501 (2013).

[38] I. Friedrich, V. Weidenhof, W. Njoroge, and M. Wuttig, J. Appl. Phys. 87, 4130 (2000).

[39] R. A. Street, G. Davies, and A. D. Yoffe, J. Non-Cryst. Solids 5, 276 (1971).

[40] See Supplemental Material at http://link.aps.org/supplemental/ 10.1103/PhysRevB.95.094111 for a comparison of impedance measurements in transmission and reflection, an example of the impact of interface effects, the verification of the device resistance's scaling behavior, and the FTIR transmission spectra.

[41] G. Chanda, R. P. S. M. Lobo, E. Schachinger, J. Wosnitza, M. Naito, and A. V. Pronin, Phys. Rev. B 90, 024503 (2014).

[42] A. B. Kuzmenko, Rev. Sci. Instrum. 76, 083108 (2005).

[43] Impedance Spectroscopy: Theory, Experiment, and Applications, edited by E. Barsoukov and J. R. Macdonald (John Wiley, Hoboken, NJ, 2005), p. 16.

[44] Z. V. Mocanu, G. Apachitei, L. Padurariu, F. Tudorache, L. P. Curecheriu, and L. Mitoseriu, Eur. Phys. J. Appl. Phys. 56, 10102 (2011). 
[45] G. Singh, A. Kaura, M. Mukul, and S. K. Tripathi, J. Mater. Sci. 48, 299 (2013).

[46] Y. H. Huang, Y. J. Huang, and T. E. Hsieh, J. Appl. Phys. 111, 123706 (2012).

[47] S. R. Elliott, Adv. Phys. 36, 135 (1987).

[48] A. M. Farid, H. E. Atyia, and N. A. Hegab, Vacuum 80, 284 (2005).

[49] J. F. Scott, Phys. Rev. B 4, 1360 (1971).
[50] X. Gonze and C. Lee, Phys. Rev. B 55, 10355 (1997).

[51] U. V. Waghmare, N. A. Spaldin, H. C. Kandpal, and Ram Seshadri, Phys. Rev. B 67, 125111 (2003).

[52] S. Mukhopadhyay, J. F. Sun, A. Subedi, T. Siegrist, and D. J. Singh, Sci. Rep. 6, 25981 (2016).

[53] H. B. Gray, Electrons and Chemical Bonding (W. A. BenjaminInc., New York, NY, 1965), p. 72. 